\title{
Reliable Transmission of Multimedia Streaming In Cluster-Based Ad Hoc Networks
}

\author{
Meng-Yen Hsieh ${ }^{1,2}$ Yueh-Min Huang ${ }^{1}$ You-Sheng Liu ${ }^{1}$ \\ ${ }^{1}$ Department of Engineering Science, National Cheng-Kung University, Taiwan, ROC \\ ${ }^{2}$ Department of Information Science, Hsing-Kuo University of Management,Taiwan, ROC
}

\begin{abstract}
Cluster-based routes help data transmission in ad hoc networks. Mobile users are organized into a cluster for requiring common multimedia service. However, node mobility does not guarantee multimedia transmission. This study presents a measure of cluster-based route's stability based on a prediction of connection probability at a specific time in future. The selected route is sufficiently stable to enable clustered mobile users to receive time-consuming multimedia streaming in future. Inter-cluster connectivity maintenance schemes, including forward and backward connectivity methods to provide non-stop transmission, maintain inter-cluster links. Finally, analytical and evaluation results indicate that the proposed route chosen based on the connection probability scheme is effective and stable for transmitting multimedia streaming in ad hoc networks.
\end{abstract}

Keywords: Cluster-based routing protocol, connection probability, link prediction, multimedia streaming.

\section{Introduction}

All Mobile ad hoc networks lead to significant challenges in transmission of multimedia streaming. For considering ad hoc multimedia applications towards group behavior in realistic scenarios, localized mobile users with on-demand request of common multimedia service are organized into a cluster to consume multimedia streaming. This study addresses a probability measure to specify the most stable clusterbased route, which does not crash during timeconsuming multimedia stream transmissions. Since multi-hop routing in ad hoc networks is unexpectedly broken, this study also provides inter-cluster connectivity maintenance including forward and backward schemes to recover broken connections.

In realistic ad hoc network applications, several schemes [1,2] model ad hoc groups with different mobility patterns, mobility rates and coverage areas, reacting on team activities and exhibiting collaborative mobility behavior. This study infers that all nodes in a cluster consuming common streams have the same mobility related to a leader node as an entry providing the service to other nodes. Due to dynamic topology, limited transmission range, and multi-hop transport, ad hoc networks must support special routing schemes with scalable and stable strategies such as on-demand routing, proxy-based routing, predicted routing, and cluster-based routing. The link prediction in [4] calculates link expiration time between any two near nodes. A routing method named as the cluster-head gateway switch routing protocol [8] has been adopted and extended into many papers. Other clustering protocols $[3,8]$ require that one of a pair of directly connected cluster leaders to relinquish its leader status. The ARC [5] provides for multiple communication paths between clusters, and implements leader maintenance to avoid the rippling effect.

This study proposes a probability measure for predicting stable and reliable transmission of multimedia streaming and finds the optimum clusterbased route for time-consuming multimedia service with forward and backward connectivity maintenance. This study considers link expiry and probability measures, and uses the ARC leader maintenance method. The simulation was implemented on the basis that a cluster consuming one multimedia stream overlappings with other clusters. The remainder of this paper is organized as follows. Section 2 introduces a system model with a cluster-based routing protocol. Section 3 then describes a probability measure of predicting connectivity in cluster-based routes before transmission of multimedia streaming. Next, Section 4 describes how two consecutive clusters supply connectivity maintenance against sudden disconnection. Section 5 discusses the analyses and evaluation results validating the prediction accuracy of multimedia transport in cluster-based ad hoc networks. Conclusions are finally drawn in Section 6.

\section{System Model}

Cluster-based routing protocol (CBRP) [3] reduces traffic overhead in routing process. CBRP has many benefits over other routing protocols, such as time efficiency and resource preservation. Each cluster 
specifies one node as a head, which is responsible for inter-cluster routing works. A gateway node bridges two or more neighboring clusters. For instance, a source node $S$ establishes a strictly cluster-based route $(S \rightarrow G \rightarrow D)$ and a loosely cluster-based route $(\mathrm{S} \rightarrow \mathrm{C} 1 \rightarrow \mathrm{C} 2 \rightarrow \mathrm{D})$ to a destination $D$ as depicted in Fig. 1.

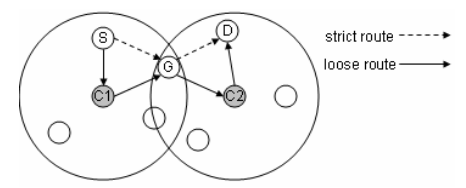

Fig. 1: A strictly and loosely cluster-based routes In this study, the loosely cluster-based route was adopted to avoid the routing overhead of repairing multimedia transmission. The leadership change mechanism of the ARC was used to demonstrate that a leader change only occurs when one cluster becomes a subset of another cluster.

If $C 1 \subset C 2$, i.e, $\forall x$, if $x \in C 1$, then $x \in C 2$, where $C 1$ and $C 2$ are different clusters.

A cluster-based multimedia service transmits multimedia streaming in ad hoc networks. We depict the service in which a cluster grouped by localized mobile consumers with common requests of multimedia service (MMS) acquires the multimedia streaming. After a cluster is formed, the head broadcasts the need for a particular multimedia file with its cluster ID (CID) as its IP to the network. When accepting the request, the multimedia provider holding the multimedia executes a cluster-based routing protocol. A routing discovery packet constructed by the provider looks for the demand cluster by flooding through all clusters. A routing reply packet constructed by the head responds to the provider along the optimum cluster-based route. The provider delivers the multimedia stream to the demand cluster when it successfully gains the route. Figure 2 illustrates an overview of the multimedia transmission from a provider, $P$, to a demand cluster, $C 4$.

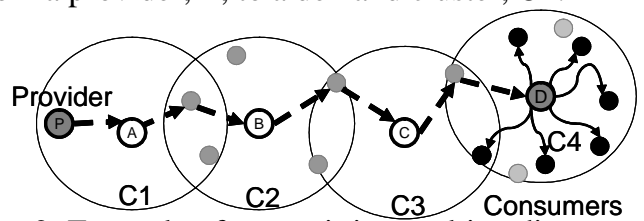

Fig. 2: Example of transmitting multimedia streaming

In this study, all multimedia sources have unique MMS identities. Hence, a demand cluster requests only one provider at any time. This design avoids collision between two or more providers carrying same multimedia at the same time.

\section{Cluster-Based Route Prediction}

A probabilistic model is provided to measure the future connection probability of any two neighboring clusters acting on a loose cluster-based route. The connectivity between two neighbor heads without direct connection depends on the existence of common gateways in their intersection. Consider two neighbor clusters overlapping with an intersection region. The intersection region is determined since all heads equip GPS devices. The predicted probability of connectivity of these clusters is derived from the size of their intersection region at a specified time in future. The connection probability is given by the Position Distribution with the mean $\lambda A$.

$$
P g(x \geq 1 \mid \lambda A)=\left\{\begin{array}{l}
1, \quad \text { if } 0 \leq d \leq r \\
1-\frac{(\lambda \times A)^{0}}{0 !} e^{-\lambda \times A}, \quad \text { if } d>r
\end{array}\right.
$$

where $r$ is the radius of a head; $d$ is the distance between two near heads; $\lambda$ is the node density; $A$ is the predicted intersection region at a specified time, and $P g$ is the predicted probability of the head. $P g_{i, j}()$ represents the probability that head $H_{i}$, finds gateways to head $H_{j}$. The equation defines the probability of connectivity of two overlapping clusters at time $t$.

$\operatorname{Pr}_{i, j}(t)=P g_{i j}\left(x_{t} \geq 1 \mid \lambda_{i}, A_{i, j}(t)\right) \times P g_{j i}\left(x_{t} \geq 1 \mid \lambda_{j}, A_{i, j}(t)\right)$ Clusters $C_{i}$ and $C_{j}$ share a intersection region $A_{i, j}(t)$ at time $t$, but have distinct node densities, $\lambda_{i}$ and $\lambda_{j}$.

The prediction of connection probability of a cluster-based route is obtained by combining all predicted probabilities of connectivity of any two consecutive clusters in the route. Figure 3 illustrates two established cluster-based routes.

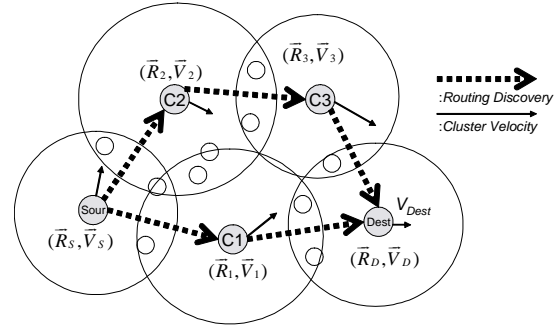

Fig. 3: Loose cluster-based routes with GPS The predicted connection probabilities of two routes, Sour $\rightarrow \mathrm{C} 1 \rightarrow$ Dest and Sour $\rightarrow \mathrm{C} 2 \rightarrow \mathrm{C} 3 \rightarrow$ Dest, at time $t$ are calculated by the following formulae.

$\operatorname{Pr}_{S, C 1, \text { Dest }}^{\text {path }}=\operatorname{Pr}_{\text {Sour }, \text { C1 }}(t) \times \operatorname{Pr}_{C 1, \text { Dest }}(t)$

$\operatorname{Pr}_{S, C 2, C 3, \text { Dest }}^{\text {path }}=\operatorname{Pr}_{\text {Sour }, C 2}(t) \times \operatorname{Pr}_{C 2, C 3}(t) \times \operatorname{Pr}_{C 3, \text { Dest }}(t)$

A novel approach, PLCBRP, combines the clusterbased routing with the connectivity prediction to construct loosely cluster-based routes. The PLCBRP creates requests or reply packets (CRREQ/CRREP). A CRREQ packet spreading through all clusters involves GPS fields containing location values and velocity vectors, a probability field for connection probability and a time field containing a future time. First, a multimedia provider (Provider) creates a CRREQ and delivers it to its head (Sour). The Provider sets a future 
time and the probability value $\langle P\rangle$ to 1 . However, the Sour sets its cluster density value $\langle\lambda\rangle$ and the GPS fields ( $\langle\vec{R}\rangle,\langle\vec{V}\rangle$ ). Since the CRREQ is attached with the address of gateways, $G_{\text {Sour }}$, bridging neighbor clusters, the Sour disperses the CRREQ. Second, a head receiving a CRREQ packet predicts the connection probability between itself and the head from which it accepted the packet. The head predicts the connection probability with its previous head. After replacing the density values and GPS information of its previous head, it continues the PLCBRP routing, modifies the gateway addresses, and delivers the changed CRREQ to its neighbor heads. Third, any head receives and processes the packet with the method as the second step, and delivers the modified CRREQ to its neighbor heads. The head of the destination cluster, denoted as Dest, accepts the packet, lastly. Fourth, the Dest waits for an appropriate time to intercept all possible arriving CRREQs. The head calculates the last connection probability between itself and its previous head in each path matching with each CRREQ. The Dest chooses the optimum cluster-based route with the maximum connection probability after comparing with the calculated probabilities of connection availability of all possible cluster-based routing paths at the same future time. Finally, the Dest replies with a CRREP packet along the decided cluster-based route as a loose source cluster-based route. After finishing the discovery phase, Sour transmits the required multimedia stream along a stable cluster-based route.

The size of the intersection region of any two overlapping clusters determines their connection probability if the heads do not connect directly each other. An intersection area is calculated from the distance of their heads $(X)$, the fan-shaped area, the triangle area, and Pythagoras' Theorem. The intersection region is given by

$\operatorname{Area}(X)=2 r^{2} \cos ^{-1}\left(\frac{X}{2 r}\right)-(X) \sqrt{r^{2}-\frac{(X)^{2}}{4}} \quad$, where two radii are equal $(r 1=r 2=r)$ and $\mathrm{r}<X$.

With $r 1, r 2 \leq X \leq(r 1+r 2)$ or $r 1<X<r 2$, the intersection region is calculated by

$\operatorname{Area}(X)=r_{1}^{2} \cos ^{-1}\left(\frac{X^{\prime}}{2 r_{1}}\right)+r_{2}^{2} \cos ^{-1}\left(\frac{X^{\prime \prime}}{2 r_{2}}\right)-X \sqrt{r_{1}^{2}-\left(\frac{X^{\prime}}{2}\right)^{2}}$, where $X^{\prime}=\frac{X^{2}+r_{1}^{2}-r_{2}^{2}}{X}$, and $X^{\prime \prime}=\frac{X^{2}+r_{2}^{2}-r_{1}^{2}}{X}$.

\section{Connectivity Maintenance}

Mobility prediction [10] enhances the on-demand routing protocols by forecasting the link expiration time between two adjacent nodes. The connectivity schemes send a maintenance packet storing the minimum link expiration time (MinLinkTime) among all hops for each delivery connection.

\section{A. Backward Connectivity Maintenance}

If a head does not accept multimedia streaming from its previous head in the cluster-based route, or if the links between the heads unexpectedly break, then the backward connectivity scheme is executed with a two-hop beacon. When the backward connectivity maintenance begins, a special beacon, denoted as BWBeacon(TTL, HeadID, NodeList, MinLinkTime) is flooded, where: Time-to-Live (TTL) is always set to 2; HeadID represents the target head; NodeList $(\mid$ NodeList $\mid \leq T T L)$ is a two-hop travel route, and MinLinkTime represents the minimum link expiration time in each two-hop route. Figure 4 illustrates the backward connectivity maintenance, where $C 1, C 2$ and C3 denote heads from Sour to Dest. Other nodes are gateways and normal nodes. Consequently, C1 retrieves and stores three two-hop routes into the intercluster routing table after obtaining three coming BWBeacons from $C 2$. After updating its' routing table, C1 transmits the streaming along the new route $(C 1 \rightarrow \underline{A} \rightarrow C 2)$, since this route has the longest connection time among three new routes.

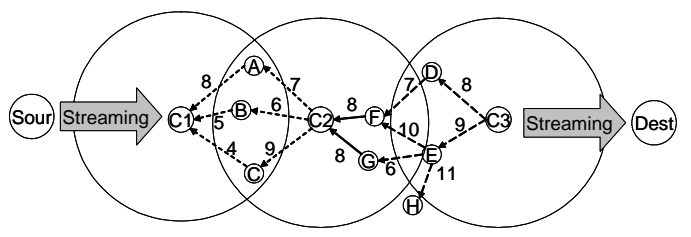

Fig. 4: BWBeacon in backward connectivity

\section{B. Forward Connectivity Maintenance}

If a head does not deliver multimedia streaming, and miss a BWBeacon from its next head, then the forward connectivity scheme is run by a two-hop routing discovery packet. An on-demand routing scheme as the DSR is adopted. However, two features are designed in this maintenance. One is that a routing request packet is set $T T L=2$. Another is that the optimum concept of DSR is used. A request packet is denoted as FWRREQ(TTL, HeadID, NodeList, MinLinkTime), and has the same four additional fields of BWBeacon. A reply packet, denoted as FWRREP(NodeList, REPTime), attends two additional fields. The NodeList value equals that of the corresponding FWRREQ plus the ID of the target head, and REPTime is the expiration time of one discovering route. Each neighboring node of the target head that accepts an FWRREQ has to reply with an FWRREP.

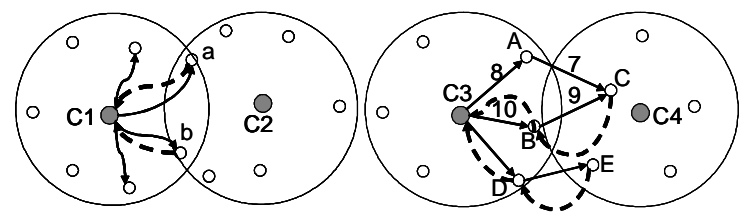

Fig. 5: FWRREQ/FWRREP in forward connectivity 
Figure 5 illustrates the maintenance. Nodes C1, $C 2, C 3$, and $C 4$ are heads. If $C 1 / C 3$ cannot transmit multimedia streams at a waiting time of $t_{\mathrm{f}}$ without accepting any BWBeacons from $C 2 / C 4$, then they flood an FWRREQ toward to the $C 2 / C 4$. Finally, $C 1$ gains two new routes $(C 1 \rightarrow a \rightarrow C 2$ and $C 1 \rightarrow b \rightarrow C 2)$, and $C 3$ gains two new routes $(C 3 \rightarrow B \rightarrow C \rightarrow C 4)$ and $(C 3 \rightarrow D \rightarrow E \rightarrow C 4)$. They select the most stable route to continue multimedia streaming.

\section{Analyses and Simulation}

We give an illustration in Figure 3. Table 1 has the initial arguments. The analyses results predicted respectively connection probabilities of these two cluster-based routes in Figure 6 after 10, 30, 60, and 90 minutes. The records are presented in Table 2. Therefore, if one multimedia application plays about one hour, the optimum route is Sour $\rightarrow \mathrm{C} 2 \rightarrow \mathrm{C} 3 \rightarrow$ Dest.

Table 1: Clusters' parameters in Figure 5

\begin{tabular}{|l|l|l|l|l|l|}
\hline Head & Sour & $\mathrm{C} 1$ & $\mathrm{C} 2$ & $\mathrm{C} 3$ & Dest \\
\hline$\vec{R}=(x, y)$ & 150,150 & 300,125 & 200,300 & 350,300 & 400,150 \\
\hline$\vec{V}=(v, \theta)$ & 1,0 & $0.8,5.23$ & $1.5,0.25$ & $1.25,523$ & $0.9,0.25$ \\
\hline Radius(mi) & 100 & 70 & 125 & 90 & 80 \\
\hline Density $\left(10^{-4}\right)$ & 3 & 4 & 3.5 & 1.5 & 3 \\
\hline
\end{tabular}

Table 2: Connection probabilities after time $t$

\begin{tabular}{|c|c|c|}
\hline Cluster-based Routes & Sour $\rightarrow \mathrm{C} 1 \rightarrow$ Dest & Sour $\rightarrow \mathrm{C} 2 \rightarrow \mathrm{C} 3 \rightarrow$ Dest \\
\hline After $10 \mathrm{~min}$ & 0.141 & 0.031 \\
\hline After $30 \mathrm{~min}$ & 0.110 & 0.128 \\
\hline After $60 \mathrm{~min}$ & 0.004 & 0.155 \\
\hline After $90 \mathrm{~min}$ & 0 & 0.038 \\
\hline
\end{tabular}

The transmission of multimedia streaming was carried out over the PLCBRP scheme. The ad hoc environment simulated in the NS2 model involves 50 mobile nodes in $1000 \mathrm{~m} \times 1000 \mathrm{~m}$ regions. The node velocity assigned to be smaller than 5 meter per second is low as the appearance of a realistic application while mobile users are focus on consuming multimedia streaming inside ad hoc clusters. The application transforms a $352 \times 288$ CIF video file [9] into one compressed file including 1000 video frames. The UDP protocol is used in the transport layer of network simulation.

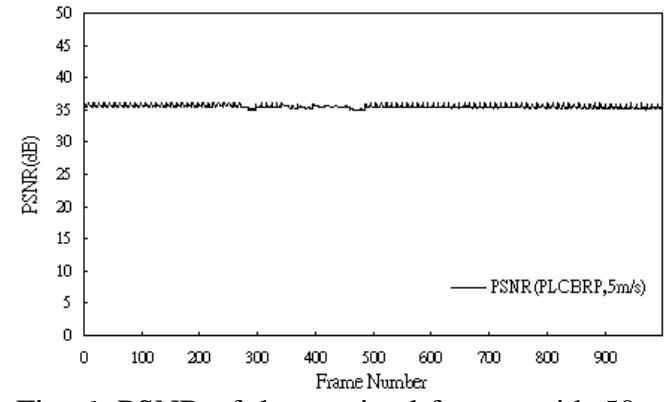

Fig. 6: PSNR of the received frames with 50 nodes at constant velocities in a $1000 \mathrm{~m} \times 1000 \mathrm{~m}$ region.

\section{Conclusions}

This study presents a model with a cluster-based multimedia service. An on-demand connection prediction to measure the likelihood of connectivity of cluster-based routes in a future time is applied to the transmission of multimedia streaming. This prediction scheme considers the intersection region of two overlapping clusters on the same cluster-based route during multimedia transmission. This study also proposes a routing method called PLCBRP, which combines the cluster-based routing protocol with the prediction scheme. For transporting nonstop multimedia streams, connectivity maintenances including forward and backward connectivity schemes with link expiration time prediction are designed to identify any existing route between two neighboring clusters. The simulation results show the PLCBRP is effective to transmit multimedia streams.

\section{References}

[1] H. Karen and Baochun Li, “Group Mobility and Partition Prediction in Wireless Ad Hoc Networks," Proc. IEEE International Conference on Communications, ICC 2002.

[2] W.T. Chen and P.Y. Chen, "Group Mobility Management in Wireless Ad Hoc Networks," IEEE VTC, Vol. 4, Oct. 2003 pp. 2202 - 2206.

[3] M. Jiang, J. Li, and Y. -C. Tay, "Cluster Based Routing Protocol Functional Specification," Internet Draft, draft-ietf-manet-cbrpspec *.txt, work in progress.

[4] Y. Yi, S.J. Lee, W. Su, and M. Gerla, On-Demand Multicast Routing Protocol (ODMRP) for Ad Hoc Networks. Internet draft, draft-ietf-manetodmrp-04.txt (Febuary 2003).

[5] E. M. Belding-Royer, "Multi-level hierarchies for scalable ad hoc routing”, Wireless Networks, 9 (2003), pp. 461--478.

[6] Royer, E.M.; Chai-Keong Toh, “A Review of Current Routing Protocols for Ad Hoc Mobile Wireless Networks,” IEEE Personal Communications, Vol. 6, pp. 46-55, April 1999.

[7] D.J. Baker and A. Ephremides, "The architectural organization of a mobile radio network via a distributed algorithm," IEEE Transaction on Communication Vol. 29, pp. 1694-1701, 1981.

[8] C.-C. Chiang, H.-K. Wu, W. Liu, and M. Gerla, "Routing in Clustered Multihop, Mobile Wireless Networks with Fading Channel," Proc. IEEE SICON'97, pp.197-211, Apr. 1997.

[9] YUV video sequence (CIF) , http://www.tkn.tuberlin.de/research/evalvid/cif.html

[10] W. Su, S.J. Lee, and M. Gerla, "Mobility prediction and routing in ad hoc networks", International Journal of Network Management, 2002. 